\title{
Composite Fermions in Modulated Structures: Transport and Surface Acoustic Waves
}

\author{
Felix von Oppen, ${ }^{1,2}$ Ady Stern, ${ }^{1}$ and Bertrand I. Halperin ${ }^{3}$ \\ ${ }^{1}$ Department of Condensed Matter Physics, The Weizmann Institute of Science, Rehovot 76100, Israel \\ ${ }^{2}$ Max-Planck-Institut für Kernphysik, Postfach 103980, 69029 Heidelberg, Germany \\ ${ }^{3}$ Physics Department, Harvard University, Cambridge, Massachusetts 02138
}

(November 12, 2018)

Motivated by a recent experiment of Willett et al. [Phys. Rev. Lett. 78, 4478 (1997)], we employ semiclassical composite-fermion theory to study the effect of a periodic density modulation on a quantum Hall system near Landau level filling factor $\nu=1 / 2$. We show that even a weak density modulation leads to dramatic changes in surface-acousticwave (SAW) propagation, and propose an explanation for several key features of the experimental observations. We predict that properly arranged $d c$ transport measurements would show a structure similar to that seen in SAW measurements.

PACS numbers: 71.10.Pm, 73.40.Hm, 73.20.Dx

Experiments on surface acoustic waves (SAW) propagation above a two-dimensional electron gas (2DEG) [1.2] provided strong support for the composite-fermion approach to the compressible quantum-Hall state near filling factor $\nu=1 / 2$ [3,, . Measurements of both absorption and velocity shift of SAW's probe the conductivity of the 2DEG at finite wavevector and frequency [1]. In this way, Willett et al. [2] observed that the absorption (velocity shift) of the SAW as function of filling factor exhibits a maximum (minimum) at $\nu=1 / 2$, implying a maximum in the conductivity. Exactly at $\nu=1 / 2$, the conductivity is found to be linear in the wavevector for wavelengths smaller than the composite-fermion mean free path, in agreement with composite-fermion theory.

Recently, Willett et al. observed a striking effect in SAW measurements near $\nu=1 / 2$ on samples whose electronic density $n(\mathbf{r})=\bar{n}+\delta n(x)$ is periodically modulated in one direction, say $\hat{x}$ [5]. When the SAW propagates in the $\hat{y}$-direction, a rather weak density modulation $(\delta n / n \leq 0.05)$ turns the minimum in the velocity shift at $\nu=1 / 2$ into a surprisingly robust maximum: Unlike the former, the magnitude and width of the latter are almost independent of the SAW wavevector $q$ and the modulation period $a$ (for sufficiently small $a$ ). In contrast, the modulation has no significant effect when the SAW propagates in the $\hat{x}$ direction.

In this Letter, we analyze $d c$ transport, SAW velocity shift and SAW absorption in modulated systems near $\nu=$ $1 / 2$. We employ semiclassical composite-fermion theory [3,6], which allows one to derive a Boltzmann equation for composite fermions (CF's). Within this theory, one attaches two Chern-Simons flux quanta to each electron.
The resulting quasi-particles - CF's - experience an effectively reduced magnetic field, $\mathcal{B}(\mathbf{r})=B-(2 h / e) n(\mathbf{r})$ so that a density modulation leads to a modulated magnetic field $\mathcal{B}(\mathbf{r})$. Thus, experiments on modulated structures test a fundamental aspect of the theory.

We first consider $d c$ transport in modulated systems with very large modulation period $a$. For such systems the current $\mathbf{J}(\mathbf{r})$ is related to the electric field $\mathbf{E}(\mathbf{r})$ by a local resistivity tensor $\rho(x)$. The measurable quantity, however, is the macroscopic resistivity $\rho^{\text {mac }}$, relating the spatially averaged current and field. We now show that the modulation makes $\rho^{\text {mac }}$ anisotropic although locally $\rho_{x x}=\rho_{y y}$. The local resistivity is a function of the local density $n(x)$ and can be written as $\rho(x) \equiv \bar{\rho}+\delta \rho(x)$, where $\delta \rho$ has zero spatial average. [Here and below, bars denote quantities in the unmodulated system]. We assume a strong magnetic field so that $\rho_{x y} \gg \rho_{x x}, \rho_{y y}$, and neglect the diagonal elements of $\delta \rho$. Since the density depends only on $x$, the current, electric field, and $\rho$ are all independent of $y$. Conservation of current then implies that $J_{x}$ is uniform in space. From Maxwell's equations, we have $\nabla \times \mathbf{E}=0$, which implies $\delta \rho_{y x} J_{x}+\rho_{y y} \delta J_{y}=0$. Here, $\delta J_{y}$ is the modulated part of the current in the $y$ direction, whose spatial average is zero. Thus [7], while $\rho_{y y}^{\mathrm{mac}}=\bar{\rho}_{y y}$

$$
\rho_{x x}^{\mathrm{mac}}=\bar{\rho}_{x x}\left(1+\beta^{2} \delta n_{r m s}^{2} / \bar{n}^{2}\right),
$$

where $\delta n_{r m s}$ is the root-mean-square value of $\delta n$, the deviation of the local electron density from its mean value $\bar{n}$, and we have defined $\beta$ by,

$$
\delta \rho_{x y} \equiv \beta \rho_{x x} \delta n / \bar{n} .
$$

In a naive Drude picture $\beta=\rho_{x y} / \rho_{x x} \gg 1$. In the quantum Hall regime, empirical observations [8] that $\rho_{x x}$ is proportional to the derivative of $\rho_{x y}$ with respect to the logarithm of the magnetic field ("resistivity law"), together with the observation that $\rho_{x y}$ is primarily determined by the filling factor $\nu$, suggest that the coefficient $\beta$ is in fact a constant, independent of the applied magnetic field, and weakly temperature dependent, of order 20 or more for high-mobility samples. Thus, a weak density modulation, while having no effect on $\rho_{y y}^{\mathrm{mac}}$, strongly enhances $\rho_{x x}^{\mathrm{mac}}$.

An SAW transmitted above a 2DEG gives rise to a "bare" electric field $\mathbf{E}_{\mathbf{q}, \omega}^{\mathrm{SAW}}$, parallel to $\mathbf{q}$, due to the piezoelectric effect in GaAs. In unmodulated systems, the 
screening response of the 2DEG leads to an absorption and a velocity shift of the SAW, proportional to the imaginary and real parts, respectively, of $\left(1+i \sigma_{\alpha \alpha} / \sigma_{m}\right)^{-1}$, where $\sigma$ is the electronic conductivity at wavevector $\mathbf{q}$ and frequency $\omega=v_{s} q$ ( $v_{s}$ being the sound velocity), $\alpha$ is the direction of $\mathbf{q}$, and $\sigma_{m}=\epsilon v_{s} / 2 \pi$, with $\epsilon$ the appropriate background dielectric constant [1, 10]. As customary, the velocity shift is given relative to its value for $\sigma_{\alpha \alpha}=\infty$. One may naively conjecture that in a modulated system $\sigma$ should be replaced by $\sigma^{\text {mac }}$. Since $\sigma_{y y}^{\mathrm{mac}} \approx \rho_{x x}^{\mathrm{mac}} /\left(\bar{\rho}_{x y}\right)^{2}$, such a conjecture (to be partially verified below) predicts a modulation-induced suppression of velocity shift when $\mathbf{q} \| \hat{y}$, and no effect when $\mathbf{q} \| \hat{x}$. In strong magnetic fields $\sigma_{m}$ is large compared to $\sigma_{\alpha \alpha}$, so that a decrease in the velocity shift corresponds to an increase in the absorption and vice versa.

The local approximation obviously applies when the modulation period $a$ is much larger than the composite fermion scattering length $\ell_{\mathrm{tr}}$. Even when this condition does not hold the resistivity $\rho$ is still local far from $\nu=1 / 2$, where the composite fermion cyclotron radius is much smaller than $a$. Thus, away from $\nu=1 / 2$ we still expect the modulation to enhance the $d c$ resistivity following Eq. (1) and similarly to enhance SAW absorption and suppress the velocity shift. Near $\nu=1 / 2$, however, we expect these effects to be greatly reduced if $a<\ell_{\mathrm{tr}}$, leading to a local minimum in the resistivity and $S A W$ absorption (as a function of magnetic field), and a maximum in the SAW velocity shift. [9] The detailed analysis we present below confirms these general expectations.

We now proceed to study SAW propagation without assuming a local resistivity tensor. The electric field $\mathbf{E}_{\mathbf{q}, \omega}^{\mathrm{SAW}}$ induces electronic currents and densities in the 2DEG. In linear response, the current $\mathbf{J}$ is related to the SAW field by $\mathbf{E}^{\mathrm{SAW}}=\rho_{\mathrm{SAW}} \mathbf{J}$. The matrix $\rho_{\mathrm{SAW}}$ differs from the electronic resistivity, $\rho$, which relates the current to the total electric field $\mathbf{E}^{\mathrm{SAw}}+\mathbf{E}^{\text {ind }}$ acting on the electrons. The field $\mathbf{E}^{\text {ind }}$ due to the induced electronic charge density can be linearly related to the current by $\mathbf{E}^{\text {ind }}=\mathcal{U} \mathbf{J}$ (with $\mathcal{U}$ given below). Clearly, $\rho_{\mathrm{SAW}}=\rho-\mathcal{U}$. In modulated systems, both $\rho$ and $\rho_{\mathrm{SAW}}$ are non-diagonal in momentum: an electric field of wavevector $\mathbf{q}$ induces currents and densities of wavevectors $\mathbf{q}+l \mathbf{p}$, where $l$ is an integer and $\mathbf{p}=2 \pi \hat{x} / a$. We use the notation $\left(\rho_{\mathrm{SAW}}(\mathbf{q})\right)_{\alpha \beta}^{k l}$ for the ratio of the field $E_{\alpha}^{\mathrm{SAW}}$ of wavevector $\mathbf{q}+k \mathbf{p}$ to an applied current $J_{\beta}$ of wavevector $\mathbf{q}+l \mathbf{p}$. The $\omega$ dependence is left implicit since all quantities are diagonal in $\omega$. Without modulation, $\rho$, $\rho_{\mathrm{SAW}}$, and their inverses are diagonal in the modulation indices $j, l$. By Coulomb's law and the continuity equation, $\mathcal{U}_{\alpha \beta}^{j l}=-i \delta_{j l} \frac{\left(q_{\alpha}+l p_{\alpha}\right)\left(q_{\beta}+l p_{\beta}\right)}{\omega} \frac{2 \pi}{\epsilon|\mathbf{q}+l \mathbf{p}|}$.

The rate of energy absorption by the electrons, $P$, is

$$
P=\operatorname{Re}\left[\mathbf{J}^{*} \cdot\left(\mathbf{E}^{\mathrm{SAW}}+\mathbf{E}^{\mathrm{ind}}\right)\right]=\operatorname{Re}\left(\sigma_{\mathrm{SAW}}\right)_{\alpha \alpha}^{00}\left|E^{\mathrm{SAW}}\right|^{2}
$$

with $\sigma_{\mathrm{SAW}}=\rho_{\mathrm{SAW}}^{-1}$. The SAW velocity shift is propor- tional to $\operatorname{Im}\left(\sigma_{\mathrm{SAW}}\right)_{\alpha \alpha}^{00}+\sigma_{m}$ 2,10]. A related formalism was independently developed in Ref. [11].

Our starting point for calculating $\sigma_{\mathrm{SAW}}$ is a Boltzmann equation describing the semiclassical dynamics of CF's in a modulated potential [6]. This equation incorporates the coupling of the CF's to the Chern-Simons fields describing the interactions of the charges with the attached flux quanta. It is valid close to $\nu=1 / 2$ where the quantum mechanics of CF's can be neglected. (All relevant length scales are assumed large compared to $1 / k_{F}$.) The Boltzmann equation is an equation for $\delta n_{\mathbf{p}}(\mathbf{r}, t)$, the deviation of the composite-fermion phase-space distribution function from its equilibrium value. Within linear response,

$$
\begin{gathered}
\left\{\partial_{t}+\mathbf{v}_{\mathbf{p}} \cdot \nabla_{\mathbf{r}}-\left(\nabla_{\mathbf{r}} V^{\mathrm{sc}}\right) \cdot \nabla_{\mathbf{p}}+e\left[\mathbf{v}_{\mathbf{p}} \times \hat{\mathbf{z}} \mathcal{B}\right] \cdot \nabla_{\mathbf{p}}\right\} \delta n_{\mathbf{p}} \\
+e \mathcal{E} \cdot \nabla_{\mathbf{p}} n_{\mathbf{p}}^{(0)}-\mathcal{I}\left[\delta n_{\mathbf{p}}-\sum_{\mathbf{p}^{\prime}} \delta n_{\mathbf{p}^{\prime}}\right]=0 .
\end{gathered}
$$

Here $V^{\mathrm{sc}}(x)$ is the self-consistent equilibrium electrostatic potential creating the modulation, $\mathbf{v}_{\mathbf{p}}$ is the velocity of a composite fermion of momentum $\mathbf{p}$ and $\mathcal{I}$ is the impurity scattering collision integral. The equilibrium value of the phase space distribution function is the Fermi-Dirac distribution $n_{\mathbf{p}}^{(0)}(\mathbf{r})=f_{\mu}\left(p^{2} / 2 m+V^{\mathrm{sc}}(x)\right)$ with a chemical potential $\mu$. The composite fermions are subject to a spatially modulated effective magnetic field $\mathcal{B}(x)$. The effective electric field $\mathcal{E}=\mathbf{E}^{\mathrm{SAw}}+\mathbf{E}^{\text {ind }}+\mathbf{E}^{\mathrm{CS}}$ is composed of the physical field $\mathbf{E}^{\mathrm{SAw}}+\mathbf{E}^{\mathrm{ind}}$, and the Chern-Simons electric field $\mathbf{E}^{\mathrm{CS}}=\left(2 h / e^{2}\right) \mathbf{J} \times \hat{\mathbf{z}}$. The modulation enters Eq. (4) through $V^{\mathrm{sc}}(x), \mathcal{B}(x)$, and the Fermi velocity. The electronic current induced by $\mathcal{E}$ is $\mathbf{J}(\mathbf{r}, t)=\sum_{\mathbf{p}} \mathbf{v}_{\mathbf{p}} \delta n(\mathbf{p}, \mathbf{r}, t)$. We emphasize that while we use composite-fermion theory, we present here only measurable electronic response functions.

The essential physics is captured by a perturbative calculation of $\sigma_{\mathrm{SAW}}$ to second order in the density modulation $\delta n$. In this calculation we consider long SAW wavelengths $q \ell_{\mathrm{tr}} \ll 1$, weak disorder $k_{\mathrm{F}} \ell_{\mathrm{tr}} \gg 1$ and $p \gg q$. We first consider the SAW wavevector to be $\mathbf{q}=q \hat{y}$, i.e., perpendicular to the modulation wavevector. We write $\rho_{\mathrm{SAW}}=\bar{\rho}_{\mathrm{SAW}}+\delta \rho$ and $\sigma_{\mathrm{SAW}}=\bar{\sigma}_{\mathrm{SAW}}+\delta \sigma$. Since the Boltzmann equation is a convenient tool for calculating $\delta \rho$ in powers of $\delta n$, we write

$$
\left(\delta \sigma_{\mathrm{SAW}}\right)_{y y}^{00} \simeq\left[\bar{\sigma}_{\mathrm{SAW}}\left(-\delta \rho+\delta \rho \bar{\sigma}_{\mathrm{SAW}} \delta \rho\right) \bar{\sigma}_{\mathrm{SAW}}\right]_{y y}^{00} .
$$

Since $\bar{\sigma}$ is diagonal in its superscripts, both the rightmost and leftmost matrices are $\left(\bar{\sigma}_{\text {SAW }}\right)^{00}$. We find $\left(\bar{\sigma}_{\text {SAW }}\right)^{00}$ using $\bar{\sigma}_{\mathrm{SAW}}=(\bar{\rho}-\mathcal{U})^{-1}$. The $x x$ element does not contribute to (5). The off-diagonal elements are larger than the $y y$ element by a factor $k_{\mathrm{F}} \ell_{\mathrm{tr}}$, and are given by $\pm \frac{\bar{\sigma}_{x y}(\mathbf{q})}{1+i \bar{\sigma}_{y y} / \sigma_{m}}$. Since $q \ell_{\mathrm{tr}} \ll 1$, we may approximate $\bar{\sigma}_{x y}(\mathbf{q})$ by its $q=0$ value. The biggest contribution to the first term in $(5)$, then, is proportional to $\delta \rho_{x x}^{00}$, which is what is measured in Weiss-oscillation measurements [7]. Its contribution here is smaller by a factor $k_{\mathrm{F}} \ell_{\mathrm{tr}}$ than that of the second term in (5). 
For the second term of (5) we use the Boltzmann equation (4) to calculate $\delta \rho$ to first order in $\delta n$. We find that as long as $k_{\mathrm{F}} a \gg 1$ (a condition well satisfied by the experimental system), the Hall components of $\delta \rho^{l 0}$ are

$$
\delta \rho_{x y}^{l 0}=-\delta \rho_{y x}^{l 0}=\bar{\rho}_{x y} \frac{\delta n_{l}}{n}
$$

with $\delta n_{l}=(1 / a) \int_{0}^{a} d x \delta n(x) \exp (i 2 \pi l x / a)$. The diagonal components $\delta \rho_{x x}^{l 0}, \delta \rho_{y y}^{l 0}$ are smaller by a factor of $k_{\mathrm{F}} \ell_{\mathrm{tr}}$. By the Onsager symmetry, $\rho_{\alpha \beta}^{0 l}(B)=\rho_{\beta \alpha}^{l 0}(-B)$ [13]. These expressions, which are obvious in the local limit $a \gg \ell_{\mathrm{tr}}$, hold irrespective of the ratio of $a$ to $\ell_{\mathrm{tr}}$.

Eqs. (5),6) and the expression for $\bar{\sigma}_{x y}^{00}$ suggest that the second term in (5) can be approximated by,

$$
\left(\delta \sigma_{\mathrm{SAW}}\right)_{y y}^{00} \simeq \sum_{l \neq 0}\left(\bar{\sigma}_{\mathrm{SAW}}\right)_{y x}^{00} \delta \rho_{x y}^{0 l}\left(\bar{\sigma}_{\mathrm{SAW}}\right)_{y y}^{l l} \delta \rho_{y x}^{l 0}\left(\bar{\sigma}_{\mathrm{SAW}}\right)_{x y}^{00}
$$

This is indeed the case, since we now show that $\left(\bar{\sigma}_{\mathrm{SAW}}\right)^{l l}$ is dominated by its $y y$ element.

The response function $\left(\bar{\sigma}_{\mathrm{SAW}}\right)^{l l}$ relates an externally applied electric field of wavevector $\mathbf{q}+l \mathbf{p}$ to a current of the same wavevector in an unmodulated system. With the expression for $\mathcal{U}$, one finds for the inverse of $\left(\bar{\sigma}_{\mathrm{SAW}}\right)^{l l}$,

$$
\left(\bar{\rho}_{\mathrm{SAW}}\right)^{l l}=\left[\begin{array}{cc}
\bar{\rho}_{x x}(l \mathbf{p})+i \frac{l p}{q \sigma_{m}} & \bar{\rho}_{x y}(l \mathbf{p})+\frac{i}{\sigma_{m}} \\
\bar{\rho}_{y x}(l \mathbf{p})+\frac{i}{\sigma_{m}} & \bar{\rho}_{y y}(l \mathbf{p})+i \frac{q}{l p \sigma_{m}}
\end{array}\right]
$$

Here we approximated $\mathbf{q}+l \mathbf{p} \simeq l \mathbf{p}$. Since $p \gg q$ and $\sigma_{m} \ll e^{2} / h$, the biggest element in $\left(\bar{\rho}_{\mathrm{SAW}}\right)^{l l}$ is the $x x$ component, and, consequently, its inverse is dominated by the $y y$ component. If $q$ is small enough such that $\left(4 h / e^{2}\right) \sigma_{m} \ll p^{2} / q k_{\mathrm{F}}$ and $\left(p v_{s}\right) /\left(q v_{\mathrm{F}}\right) \gg 1$ (with $v_{\mathrm{F}}$ the composite fermion Fermi velocity), then $\left(\bar{\sigma}_{\mathrm{SAW}}\right)_{y y}^{l l} \approx$ $1 / \bar{\rho}_{y y}(l \mathbf{p}) \approx\left(e^{2} / 2 h\right)^{2} / \bar{\sigma}_{x x}(l \mathbf{p})$. The largeness of the $y y$ element, $\left(\bar{\sigma}_{\mathrm{SAW}}\right)_{y y}^{l l}$, which plays an important role in our calculation, is in marked contrast to the conductivity matrix $\bar{\sigma}^{l l}$, whose largest elements are the off-diagonal ones, due to the strong magnetic field. This contrast reflects the fact that in the modulated system, the SAW field $\mathbf{E}^{\mathrm{SAW}}$ in the $\hat{y}$ direction is accompanied by a large induced field in the $\hat{x}$ direction. The current is almost perpendicular to the total electric field and hence has a sizable component in the $\hat{y}$-direction.

Finally, using (7) and (8), we find

$$
\left(\delta \sigma_{\mathrm{SAW}}\right)_{y y}^{00} \simeq \sum_{l \neq 0}\left(\frac{\delta n_{l}}{n} \frac{e^{2} / 2 h}{1+i \bar{\sigma}_{y y} / \sigma_{m}}\right)^{2} \frac{1}{\bar{\sigma}_{x x}(l \mathbf{p})} .
$$

This is the central result of our analytical calculation. In the local limit, $p \ll 1 / \ell_{\mathrm{tr}}$, this expression can be obtained from the analysis described above Eq. (1). An analogous calculation shows that the effect for SAW propagation parallel to the modulation direction, $q=q \hat{x}$, is smaller by a factor of order $\left(k_{\mathrm{F}} \ell_{\mathrm{tr}}\right)^{2} \sim 10^{3}-10^{4}$. Experimentally, indeed, the modulation has no observable effect in this case. The modulation contribution to the macroscopic conductivity is given by a similar analysis with $\sigma_{m}=\infty$.

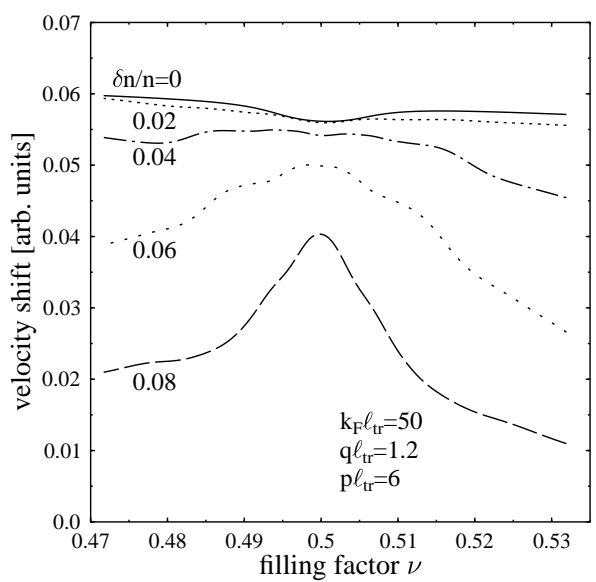

FIG. 1. SAW velocity shift vs filling factor for different modulation strengths. The SAW wavevector is $q \ell_{\mathrm{tr}}=1.2$ and the modulation wavevector $p \ell_{\mathrm{tr}}=6$. With increasing modulation, the minimum in velocity shift changes into a maximum, consistent with the experimental results of Ref. [5].

We now show that the predictions of Eq. (9) are in qualitative agreement with key experimental results. The influence of the modulation is essentially determined by $\bar{\sigma}_{x x}(l \mathbf{p})$. This quantity is known, both from previous experiments [2] and from theory [3, 边, to exhibit a maximum as a function of magnetic field at $\nu=1 / 2$ when $l|\mathbf{p}| \gg 1 / \ell_{\mathrm{tr}}$. Thus Eq. (9) predicts that the modulationdependent contribution to the absorption (velocity shift) has a minimum (maximum) around $\nu=1 / 2$, once the modulation period $a$ is smaller than $\ell_{\operatorname{tr}}$. The trends of the modulation-independent contribution, $\bar{\sigma}_{y y}^{00}$, around $\nu=1 / 2$ are just opposite. For strong enough modulation, it is the modulation-dependent contribution which determines the type of extremum points at $\nu=1 / 2$, in agreement with the new effect observed by Willett et al.

The analytical results are well supported by our numerical solutions of the Boltzmann equation, which are not restricted to weak modulation or to the regime $q \ll$ $p, \ell_{\mathrm{tr}}^{-1}$. Numerically, we directly compute the response to the applied SAW field. We restrict ourselves to a modulated magnetic field with a single Fourier component, and employ the isotropic relaxation-time approximation 12 to account for impurity scattering. Representative results for the SAW velocity shift as function of filling factor around $\nu=1 / 2$ are shown in Fig. 1. At zero modulation, the velocity shift exhibits the usual minimum at $\nu=1 / 2$. As the modulation is increased, the minimum disappears and a maximum develops in accord with the analytical conclusions above. The effect of the modula- 
tion gets stronger as one gets farther from $\nu=1 / 2$. As seen in Fig. 1, the modulation induced peak in the velocity shift is more pronounced than the minimum observed at zero modulation, in agreement with the experiment. We emphasize that we found similar behavior over a wide range of the parameters $q$ and $p$.

Experimentally, the modulation-induced peak in velocity shift was strikingly insensitive to the SAW wavevector $q$ and the modulation wavevector $p$. Fig. 2 shows our results for that peak for realistic values of $q$ and $p$. Clearly, the width and magnitude of the peak are rather stable over a substantial parameter range - a factor 2 in modulation period and a factor 3 in SAW wavelength, in good qualitative agreement with the experiment.

There is a point of disagreement between our theory and the experiment. Theoretically, the maximum in the velocity shift is primarily due to a decrease in the velocity shift away from $\nu=1 / 2$, rather than to an increase in its value at $\nu=1 / 2$. Experimentally, there seems also to be a sharp increase in $\sigma_{y y}^{00}$ at $\nu=1 / 2$. This may be caused by unknown physical effects that were omitted from our model. However, another conceivable explanation for this difference within our model might be that the material parameter $\sigma_{m}$ increases with the voltage applying the modulation, leading to an absolute increase in the velocity shift at $\nu=1 / 2$.

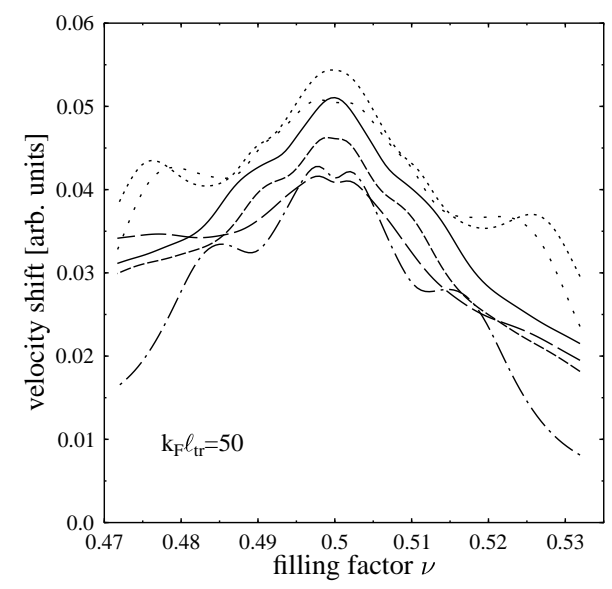

FIG. 2. Modulation-induced maximum in the SAW velocity shift vs filling factor for different SAW and modulation wavevectors $q$ and $p$. The parameters $\left(q \ell_{\mathrm{tr}}, p \ell_{\mathrm{tr}}, \delta n / n\right)$ are for the full line $(0.6,6,0.06)$, short dotted $(0.6,10,0.07)$, short dashed $(1.2,6,0.07)$, wide dotted $(1.2,10,0.08)$, long dashed $(2,6,0.08)$, and dash-dotted $(2,10,0.11)$.

In fact, the values of $\sigma_{m}$ which were used to relate SAW propagation to $\sigma$ in unmodulated samples have been much larger than the theoretical values, for reasons which are not understood [3]. Thus a dependence on the gate voltage, and perhaps on the direction of SAW prop- agation, is not inconceivable. An increase in the mean free path $\ell_{\mathrm{tr}}$ for increasing gate voltage in the modulated samples would also give qualitatively similar effects.

Our calculation for $d c$ transport, whose result was stated below Eq. (9), predicts anisotropies in the macroscopic $d c$ conductivity and resistivity tensors. Specifically, we find that both $\rho_{x x}^{\mathrm{mac}}$ and $\sigma_{y y}^{\mathrm{mac}}$ exhibit minima near $\nu=1 / 2$ (with the modulation in the $x$ direction), observable in Hall-bar and Corbino geometry, respectively. Transport experiments reported in Ref. [5] have not shown these effects. However, it is not clear what are the actual current paths in this experiment. Very recent experimental results of Smet et al. [14 are in qualitative agreement with our theory.

In conclusion, we find within a semiclassical compositefermion approach that a weak density modulation can dramatically affect both $d c$ transport properties and SAW propagation near $\nu=1 / 2$. Our results are in agreement with many key features of the experimental results. Details of the calculation will be published elsewhere.

We acknowledge financial support from the US-Israel Binational Science Foundation (AS and BIH, 95-250/1), the Minerva foundation (AS and $\mathrm{FvO}$ ), and NSF grant DMR94-16190 (BIH). We thank B. Elattari for collaboration in early stages of this work, O. Entin, Y. Levinson, J. Smet, and R.L. Willett for instructive discussions of unpublished results.

[1] R.L. Willett, Surf. Sci. 305, 76 (1994).

[2] R.L. Willett, R.R. Ruel, K.W. West, and L.N. Pfeiffer, 71, 3846 (1993).

[3] B.I. Halperin, P.A. Lee, and N. Read, Phys. Rev. B 47, 7312 (1993).

[4] B.I. Halperin, in Novel Quantum Liquids in LowDimensional Semiconductor Structures, edited by S. Das Sarma and A. Pinczuk (Wiley, New York, 1996).

[5] R.L. Willett, K.W. West, and L.N. Pfeiffer, Phys. Rev. Lett. 78, 4478 (1997).

[6] S.H. Simon and B.I. Halperin, Phys. Rev. B 48, 17368 (1993); A. Stern and B.I. Halperin, Phys. Rev. B 52, 5890 (1995).

[7] R. Menne and R.R. Gerhardts, cond-mat/9709072.

[8] H. L. Stormer et al., Sol. Stat. Comm. 84, 95 (1992). S.H. Simon and B.I. Halperin, Phys. Rev. Lett. 73, 3278 (1994).

[9] By contrast, when the modulation becomes too strong, the local composite fermion cyclotron radius will be small (compared to $a$ ) even when the average filling factor is $1 / 2$. Then, the conductivity is local even at $\nu=1 / 2$ and the structure at $\nu=1 / 2$ should disappear. This is consistent with the obervations of Ref. 沟.

[10] S. Simon, Phys. Rev. B 54, 13878 (1996).

[11] Y. Levinson et al., private communication and cond- 
mat/9712276.

[12] Our numerical results are largely insensitive to the issues raised in A. Mirlin and P. Wölfle, Phys. Rev. Lett. 78, 3717 (1997).

[13] L.D. Landau and E.M. Lifshitz Statistical Physics, sec. 122, Pergamon Press (1978).

[14] J. Smet, K. von Klitzing, D. Weiss, and W. Wegscheider, (to be published). 